Research Paper

\title{
Blood leukocytes methylation levels analysis indicate methylated plasma test is a promising tool for colorectal cancer early detection
}

\author{
Zhiliang Chen ${ }^{1,2+}$; Guodong Zhao ${ }^{3,4,6+}$; Kai Wang3; Xiaomei Wang3; Yong Ma3,5; Shangmin Xiong ${ }^{3,6}{ }^{\varpi,}$ \\ Minxue Zheng ${ }^{3,5}$; Sujuan Fei ${ }^{1,2}$ \\ 1. Department of Gastroenterology, Affiliated Hospital of Xuzhou Medical University, Xuzhou Jiangsu 221002, China \\ 2. Institute of Digestive Diseases, Xuzhou Medical University, Xuzhou Jiangsu 221002, China \\ 3. Zhejiang University Kunshan Biotechnology Laboratory, Zhejiang University Kunshan Innovation Institute, Kunshan Jiangsu 215300, China \\ 4. State Key Laboratory of Bioelectronics, School of Biological Science and Medical Engineering, Southeast University, Nanjing 210009, China \\ 5. Suzhou Institute of Biomedical Engineering and Technology, Chinese Academy of Sciences, Suzhou Jiangsu 215163, China \\ 6. Suzhou VersaBio Technologies Co. Ltd., Kunshan Jiangsu 215300, China \\ † These authors contributed equally to this work.
}

$\triangle$ Corresponding author: Shangmin Xiong; Email: shangmin_xiong@hotmail.com; Address: Zhejiang University Kunshan Biotechnology Laboratory, Zhejiang University Kunshan Innovation Institute, Kunshan Jiangsu 215300, China. Minxue Zheng; Email: minxue.zheng@sibet.ac.cn; Address: Suzhou Institute of Biomedical Engineering and Technology, Chinese Academy of Sciences, 88 Keling Road, SND, Suzhou 215000, China. Sujuan Fei; Email: xyfyfeisj99@163.com; Address: Department of Gastroenterology, Affiliated Hospital of Xuzhou Medical University, Xuzhou Jiangsu 221002, China.

(C) The author(s). This is an open access article distributed under the terms of the Creative Commons Attribution License (https://creativecommons.org/licenses/by/4.0/). See http://ivyspring.com/terms for full terms and conditions.

Received: 2020.12.13; Accepted: 2021.04.22; Published: 2021.04.30

\begin{abstract}
Background: A number of plasma methylated DNA biomarkers related to colorectal cancer (CRC) have been identified. However, the effect of methylation level in leukocytes on plasma-based methylation test was rarely reported.

Methods: Blood samples from 213 individuals including 91 CRC patients were collected and separated into $3.5 \mathrm{~mL}$ of plasma and paired leukocyte fractions. DNA were extracted from plasma and leukocytes and bisulfite converted, followed by ColoDefense test that detects methylated SEPT9 (mSEPT9) and methylated SDC2 (mSDC2) simultaneously in a single qPCR reaction.

Results: Both mSEPT9 and mSDC2 levels in leukocytes exhibited no significant difference among CRC, benign tumors and healthy controls. However, mSEPT9 and mSDC2 levels in plasma were significantly higher in CRC group than those in other groups. The sensitivities of mSEPT9 and mSDC2 alone for detecting CRC with plasma samples were $75.8 \%$ and $60.4 \%$ with specificities of $94.7 \%$ and $86.8 \%$, respectively. These two markers in combination exhibited an improved sensitivity of $85.7 \%$ for CRC detection with a specificity of $86.8 \%$, mostly attributable to increased sensitivity of $81.8 \%$ for detecting stage 0 -II CRC. AUC values for mSEPT9 and mSDC2 alone were 0.864 (95\% Cl: $0.798-0.929)$ and 0.796 ( $95 \% \mathrm{Cl}: 0.719-0.874)$, respectively, but improved to $0.972(95 \% \mathrm{Cl}: 0.949-0.996)$ when combined for ColoDefense test.

Conclusions: The leukocytes gDNA will not affect the performance of plasma ColoDefense test, and plasma ColoDefense test exhibited high sensitivity and specificity in a validation set, demonstrating its potential as a non-invasive and cost-effective method for CRC early detection.
\end{abstract}

Key words: Colorectal cancer, plasma, leukocyte, mSEPT9, mSDC2, early detection

\section{Introduction}

Liquid biopsy, primarily blood test, provides potential non-invasive screening approaches to assess many diseases including cancers. Peripheral blood is a rich library of cells and genetic materials, capable of providing real-time information originating from primary or metastatic tumor sites. The major analytes 
for identifying and quantifying genetic and epigenetic information in peripheral blood liquid biopsy include circulating cell-free DNA (cfDNA), circulating tumor cells (CTC), messenger RNA (mRNA), microRNA (miRNA) and leukocytes, etc. Each type of analyte has its own potentials and drawbacks in addressing specific needs. For example, CTCs provide intact cancer-specific information at genome, RNA and protein levels, making them a powerful tool to detect cancers in advanced stages and a reliable prognostic biomarker [1]. However, its rareness in early stage cancer and high cost in enrichment, detection and characterization made it a less prevailing screening tool in the near future.

Among these analytes, cfDNA biomarker testing is one of the most functional and promising technologies in screening, diagnosis and prognosis of cancers and other diseases. For healthy individuals or patients in the early stage of tumorigenesis, the amount of cfDNA is estimated to be approximately 3-9 $\mathrm{ng} / \mathrm{mL}$ of plasma, while there could be a 10-fold increase in the concentration of plasma cfDNA for patients with advanced cancer [2]. Main origins of cfDNA include endogenous DNA from cellular structures in circulation or nervous system that contain or release DNA during normal or abnormal activities into circulation, and exogenous DNA entering the human body via infection, ingestion, inhalation, transplant, transfusion, or as fetal DNA fragments, etc. The interaction of these activities may further stimulate the release of cfDNA in circulation [3]. Of these sources, blood cells, especially leukocytes, contribute a large amount of cfDNA in healthy subjects [4].

However, recent studies have also shown that considerable proportion of somatic mutations identified in leukocyte genomic DNA (gDNA) in individuals with or without cancer were also present in cfDNA, raising the possibility of false positive in detection of cancer-derived mutations [5] [6]. In addition, DNA and RNA sequence analysis indicated that somatic mutations exist across normal tissues in varying degrees, and genetic clones containing such mutations may not have the potential to develop into cancers [7], the widespread presence of mutations in normal tissues and cancerous lesions makes them a less valuable biomarker candidate in early cancer detection. Aberrant methylation of specific genes has been shown to be associated with many cancer types including CRC [8]. A number of abnormally methylated genes related to CRC have been identified, including methylated SEPT9, SDC2, CLIP4 and SFRP2, some of which have been commercialized for CRC screening [9-14]. SEPT9 is the coding gene for septin-9, a protein involved in cytokinesis, and its hypermethylation in CRC makes it a useful biomarker. SDC2 encodes syndecan-2, a glycoprotein involved in cell binding, signaling and cytoskeletal organization. Previous studies of methylated SDC2 $(\mathrm{mSDC})$ showed it to be a promising biomarker for both stool- and plasma-based CRC screening test [14-16]. Recently, a new plasma-based methylation test (ColoDefense test) for CRC early detection was reported, which contained both methylated SEPT9 (mSEPT9) and mSDC2 simultaneously in a single qPCR reaction with high sensitivity and specificity [14]. However, whether the plasma methylated DNA derived from leukocyte gDNA will cause the false positive in CRC detection or not was rarely reported. Here, we report the results of a study of the methylation levels of SEPT9 and SDC2 in plasma and leukocytes of patients with CRC or precancerous lesions and health subjects. Our primary goal was to evaluate the effect of methylation levels for SEPT9 and SDC2 in leukocytes on the performance of ColoDefense test, and our secondary aim was to further validate the plasma test for CRC early detection in a validation set.

\section{Materials and Methods}

\subsection{Sample collection}

Blood specimens were collected at the Affiliated Hospital of Xuzhou Medical University from January 1, 2020 to August 31, 2020. The inclusion criteria consisted of the following: aged 18 or older, no history of CRC, no pregnant woman, having colonoscopy results, and participants with abnormal colonoscopy results should have pathological diagnosis results. The exclusion criteria were as follows: missing or incomplete sample information, insufficient blood volume, repeated sampling, severe hemolysis, and insufficient DNA indicated by low ACTB levels (see data analysis). Finally, 213 blood samples were collected, including 91 from CRC patients, 49 from adenomatous polyp patients (Ade), 27 from hyperplastic polyp patients (HP), 38 from control subjects with normal colonoscopy results and 8 from patients with other gastrointestinal tumors (Figure 1). Ten milliliter blood was drawn from each subject and stored at $4^{\circ} \mathrm{C}$ for no more than 24 hours. The blood samples were centrifuged at $1,350 \mathrm{~g}$ for $12 \mathrm{~min}$ to separate plasma and leukocytes. The $3.5 \mathrm{~mL}$ plasma fractions and leukocyte fractions were then frozen at $-80^{\circ} \mathrm{C}$ until use. This study was approved by the Institutional Review Board of the Affiliated Hospital of Xuzhou Medical University (Ethics Committee reference number: XYFY2020-KL123-01), and informed consent was obtained from all participating patients and control subjects. 


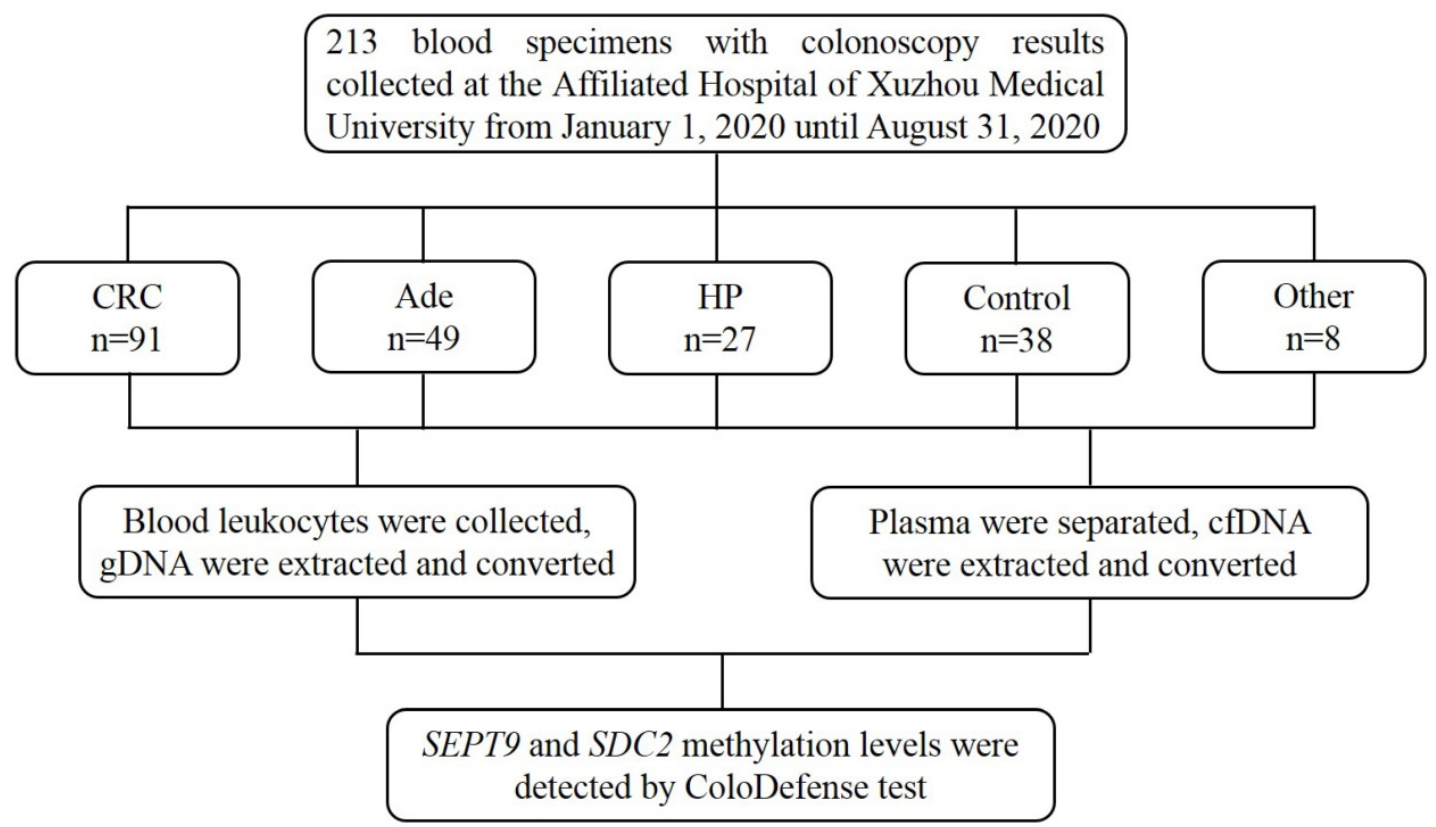

Figure 1. The flow chat of this study.

\subsection{DNA extraction and bisulfite conversion}

Leukocyte genomic DNA was isolated with VersaPrep DNA extraction kit (Suzhou VersaBio Technologies Co. Ltd., Kunshan, Jiangsu, China). Specifically, $250 \mu \mathrm{L}$ lysis buffer and $20 \mu \mathrm{L}$ proteinase $\mathrm{K}$ solution were added to each leukocyte specimen and followed by incubation at room temperature for 10 min. Afterwards, $180 \mu \mathrm{L}$ ethanol was added into each sample and the mixture was loaded onto a spin column. After two washing steps, the purified genomic DNA was eluted with $100 \mu \mathrm{L}$ elution buffer. Plasma circulating free DNA (cfDNA) was extracted using a cfDNA extraction kit (Suzhou VersaBio Technologies Co. Ltd.) according to previously published procedure [14]. Subsequently, $100 \mu \mathrm{L}$ purified cfDNA and leukocyte gDNAwere used for bisulfite conversion and purification by a fast bisulfite conversion kit (Suzhou VersaBio Technologies Co. Ltd.) [14].

\subsection{Quantitative real-time PCR}

Converted and purified DNA from leukocytes and plasma were examined by ColoDefense test (Suzhou VersaBio Technologies Co. Ltd.), a multiplex qPCR assay detecting $\mathrm{mSEPT9}, \mathrm{mSDC} 2$ and $A C T B$ simultaneously in one qPCR reaction [14]. Each plasma cfDNA sample was tested in three qPCR replicates, and a single $\mathrm{qPCR}$ reaction was performed for each leukocyte gDNA sample. The qPCR was performed on LC480-II thermal cycler (Roche Diagnostics) with the following cycling conditions: activation at $95^{\circ} \mathrm{C}$ for 30 minutes, 50 cycles of $95^{\circ} \mathrm{C}$ for 10 seconds, $58^{\circ} \mathrm{C}$ for 30 seconds, $72^{\circ} \mathrm{C}$ for 10 seconds, and final cooling to $40^{\circ} \mathrm{C}$ for 30 seconds [14].

\subsection{Data analysis}

For a plasma sample, the result was considered 'invalid' if $A C T B C t$ was greater than 35.0, and mSEPT9 and mSDC2 were considered 'detected' if their $\mathrm{Ct}$ values were less than 45.0 and 50.0, respectively. $\mathrm{mSEPT9}$ and $\mathrm{mSDC} 2$ were scored positive respectively by $1 / 3$ and $2 / 3$ rules. A plasma sample would be scored positive for ColoDefense test if either $\mathrm{mSEPT9}$ or $\mathrm{mSDC} 2$ was positive [14]. $\triangle \mathrm{Ct}$ was used to determine the methylation levels of SEPT9 and $S D C 2$ genes in leukocytes. $\triangle \mathrm{Ct}$ was defined as the difference between the $\mathrm{Ct}$ values of the target (mSEPT9 or $\mathrm{mSDC2}$ ) and the internal control gene (ACTB) [12]. Mean Ct values of mSEPT9 and mSDC2 were used to determine the methylation levels in plasma samples. For $\mathrm{qPCR}$ reactions without detectable amplification signals, their $\mathrm{Ct}$ values were set at 50, the maximal number of PCR cycles. Mean Ct values of $\mathrm{mSEPT} 9$ and $\mathrm{mSDC} 2$ for CRC patients and control subjects were also used to plot ROC curves. Data were subjected to statistical analysis with IBM SPSS software for Windows Version 22.0. $t$-test was used for comparison of two groups, and Pearson chi-square test was used for comparison among more than two groups. The differences of methylation levels were analyzed by Mann-Whitney U test.

\section{Results}

The characteristics of 213 subjects enrolled in this study are shown in Table 1 . The mean age of Ade and HP patients was 54.6 and 52.5 with $79.6 \%$ and $59.3 \%$ 
males, respectively. Ninety-one CRC patients were enrolled including 4 stage 0, 9 stage I, 31 stage II, 29 stage III, 4 stage IV and 14 of unknown stage. Other tumor groups included 5 rectal neuroendocrine tumor patients, two rectal melanoma patients and one gastric stromal tumor patients.

As shown in Figure 2, mSEPT9 (Figure 2a) and $\mathrm{mSDC2}$ (Figure 2b) levels in blood leukocytes from CRC, Ade, HP, other tumor and control groups exhibited no significant difference. In contrast, mSEPT9 and mSDC2 levels in CRC plasma were significantly higher when compared to those of Ade, $\mathrm{HP}$, other tumor and control groups, which showed no significant difference among themselves (Figures $2 \mathrm{c}$ and $2 \mathrm{~d}$ ).

Moreover, blood leukocyte $\mathrm{mSEPT9}$ and $\mathrm{mSDC} 2$ levels both showed no significant difference among CRC patients of different stages (Figures $3 a, 3 b$ ). For plasma samples, both SEPT9 and SDC2 showed higher methylation levels in stages II-III CRC patients when compared to stage 0 patients (Figures $3 \mathrm{c}$ and 3d). Furthermore, stage IV patients also displayed higher mSEPT9 level than patients of other stages (Figure 3c), but such a trend was not observed for mSDC2 levels (Figure 3d).

Table 1.The characteristics of the subjects enrolled in this study.

\begin{tabular}{|c|c|c|c|c|c|}
\hline \multirow[t]{2}{*}{ Group } & \multirow{2}{*}{$\begin{array}{l}\text { Total } \\
\text { number }\end{array}$} & \multicolumn{2}{|c|}{ Gender (\%) } & \multicolumn{2}{|l|}{ Age } \\
\hline & & Male & Female & $\begin{array}{l}\text { Mean } \\
(\mathrm{SD})\end{array}$ & $\begin{array}{l}\text { Min-M } \\
\text { ax }\end{array}$ \\
\hline Ade & 49 & $39(79.6)$ & $10(20.4)$ & $54.6(11.7)$ & $29-78$ \\
\hline $\mathrm{HP}$ & 27 & $16(59.3)$ & $11(40.7)$ & $52.5(11.2)$ & $27-77$ \\
\hline CRC & 91 & $55(60.4)$ & $36(39.6)$ & $62.7(13.9)$ & $22-89$ \\
\hline 0 & 4 & $0(0.0)$ & $4(100.0)$ & $70.3(10.4)$ & $53-80$ \\
\hline I & 9 & $3(33.3)$ & $6(66.7)$ & $56.3(12.3)$ & $30-72$ \\
\hline II & 31 & $23(74.2)$ & $8(25.8)$ & $60.9(12.8)$ & $36-85$ \\
\hline III & 29 & $18(62.1)$ & $11(37.9)$ & $64.5(12.7)$ & $25-89$ \\
\hline IV & 4 & $3(75.0)$ & $1(25.0)$ & $62.5(10.2)$ & $55-80$ \\
\hline Unknown & 14 & $9(64.3)$ & $5(35.7)$ & $64.7(18.4)$ & $22-86$ \\
\hline Other tumors & 8 & $4(50.0)$ & $4(50.0)$ & $49.6(9.6)$ & $38-68$ \\
\hline $\begin{array}{l}\text { Rectal neuroendocrine } \\
\text { tumor }\end{array}$ & 5 & $3(60.0)$ & $2(40.0)$ & $45.4(6.8)$ & $38-58$ \\
\hline Rectal melanoma & 2 & $1(50.0)$ & $1(50.0)$ & $56.5(11.5)$ & $45-68$ \\
\hline Gastric stromal tumor & 1 & $0(0.0)$ & $1(100.0)$ & $57(/)$ & / \\
\hline Control & 38 & $20(52.6)$ & $18(47.4)$ & $47.2(14.5)$ & $20-81$ \\
\hline
\end{tabular}

SD, standard deviation.
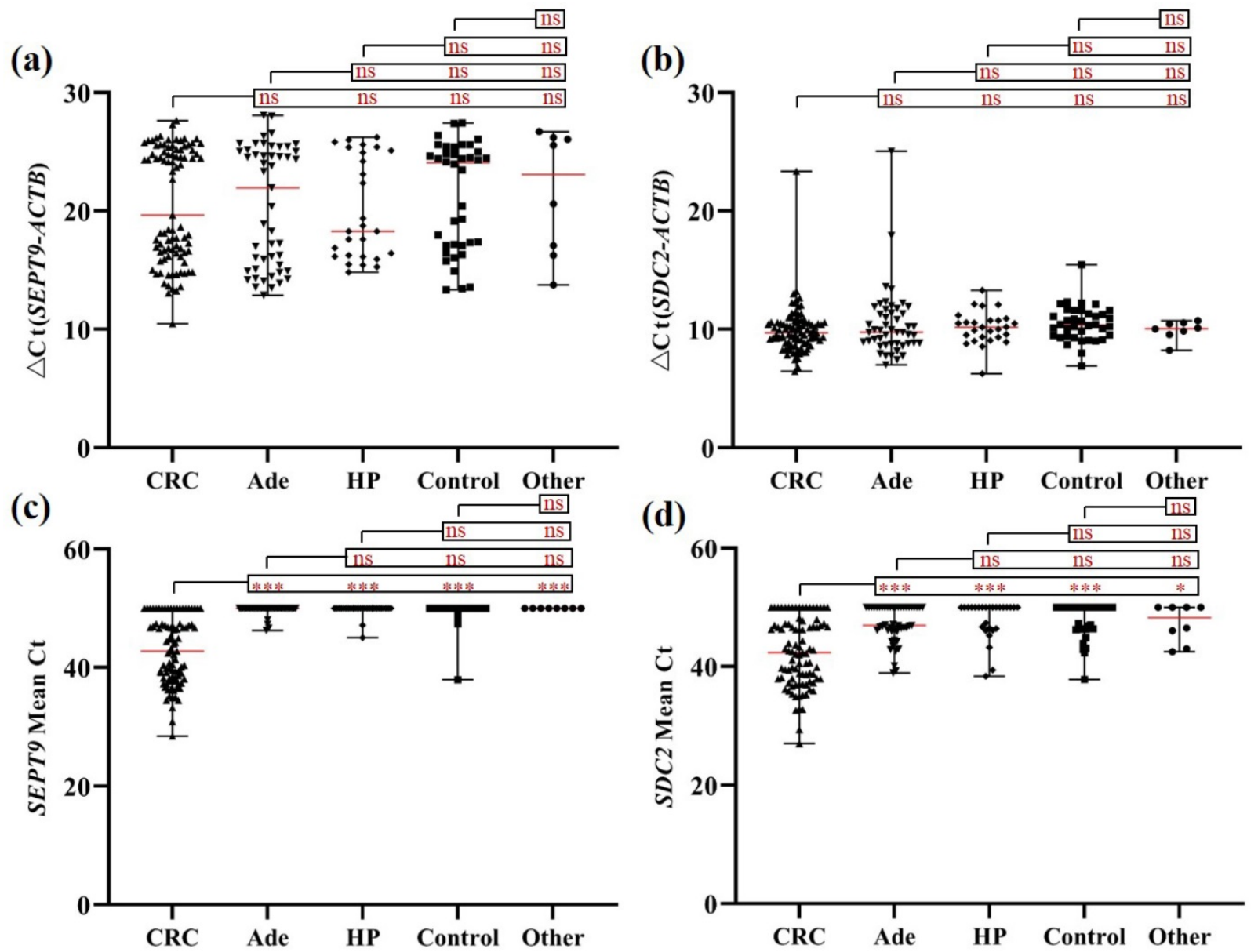

Figure 2. mSEPT9 ( $a, c)$ and mSDC2 (b, d) levels in blood leukocytes (a, b) and plasma (c, d) from CRC, Ade, HP, and other tumor patients as well as control subjects. ns, not significant. *, $p<0.05$.**, $p<0.01$. ***, $p<0.001$. Red lines represent the median methylation levels of SEPT9 and SDC2. 

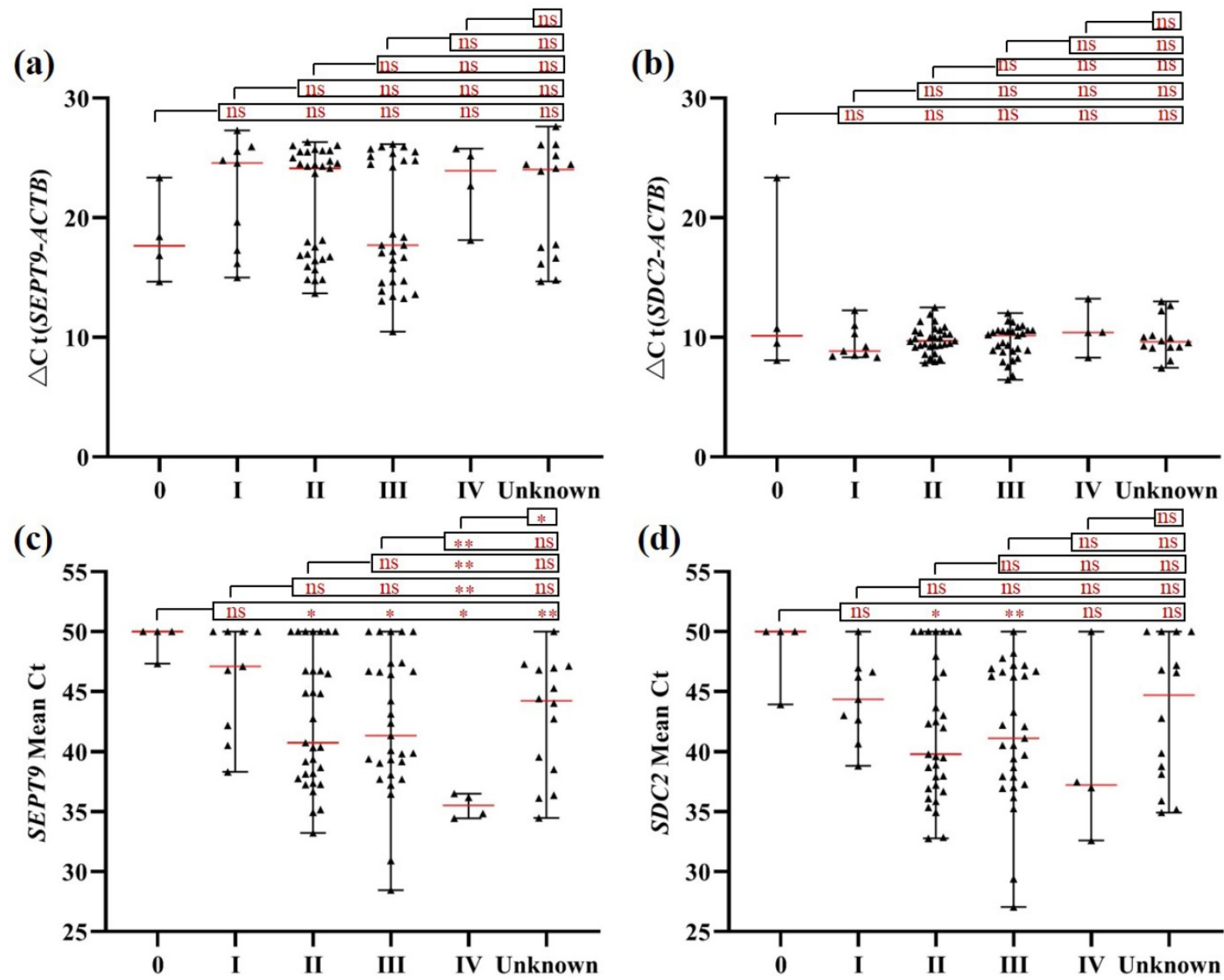

Figure 3. mSEPT9 (a, c) and mSDC2 (b, d) levels in blood leukocytes (a, b) and plasma (c, d) from CRC patients of different stages. ns, not significant. *, $p<0.05$. **, $p<0.01$. ***, $p<0.001$. Red lines represent the median methylation levels of SEPT9 and SDC2.

This study also displayed a validation results for plasma ColoDefense test in detecting CRC. The sensitivities of $\mathrm{mSEPT} 9$ alone and $\mathrm{mSDC} 2$ alone for CRC detection were $75.8 \%$ and $60.4 \%$ with specificities of $94.7 \%$ and $86.8 \%$, respectively. When mSEPT9 and mSDC2 were combined as in ColoDefense test, the sensitivity was improved to $85.7 \%$ with a specificity of $86.8 \%$ (Figure 4 ). The sensitivity improvement was mainly due to higher positive detection rates of early stage (stage 0-II) CRCs. The sensitivities of mSEPT9 alone and mSDC2 alone for stage 0 -II CRC detection were $68.2 \%$ and $61.3 \%$, but ColoDefense test showed a $81.8 \%$ sensitivity, representing $13.6 \%$ and $20.5 \%$ increase over those of each biomarker alone. The sensitivities of ColoDefense test for detecting Ade, HP and other gastrointestinal tumors were $24.5 \%, 22.2 \%$ and $25.0 \%$, respectively, which were significantly lower than that for CRC detection (Figure 4).

Finally, receiver operating characteristic (ROC) curve analysis was performed with plasma test results. Area under the curve (AUC) value for
ColoDefense test in detecting CRC was 0.972 (95\% CI: 0.949 - 0.996), whereas AUC values for $\mathrm{mSEPT}$ alone and $\mathrm{mSDC} 2$ alone were 0.864 (95\% CI: $0.798-0.929)$ and 0.796 (95\% CI: $0.719-0.874)$, respectively, 0.108 and 0.176 lower when compared to the combined ColoDefense test (Figure 5). The sensitivities of mSEPT9 alone, mSDC2 alone and ColoDefense test for detecting CRC of different characteristics exhibited no significant difference among different age groups, genders, $\mathrm{T}$ or Nstages, tumor sizes, tumor sites and degrees of differentiation. The only exception was that the sensitivities of MSEPT9 alone showed significant difference for different $\mathrm{T}$ stages (Table 2), which was likely due to the fact that $\mathrm{T}$ stage represents the size and location of primary tumors so that early T stage CRCs usually have smaller sizes and thus release less DNA into the circulation, resulting in lower sensitivity. However, adding $\mathrm{mSDC} 2$ detection to the test could compensate for the low performance of single $\mathrm{mSEPT} 9$ biomarker in this aspect. 


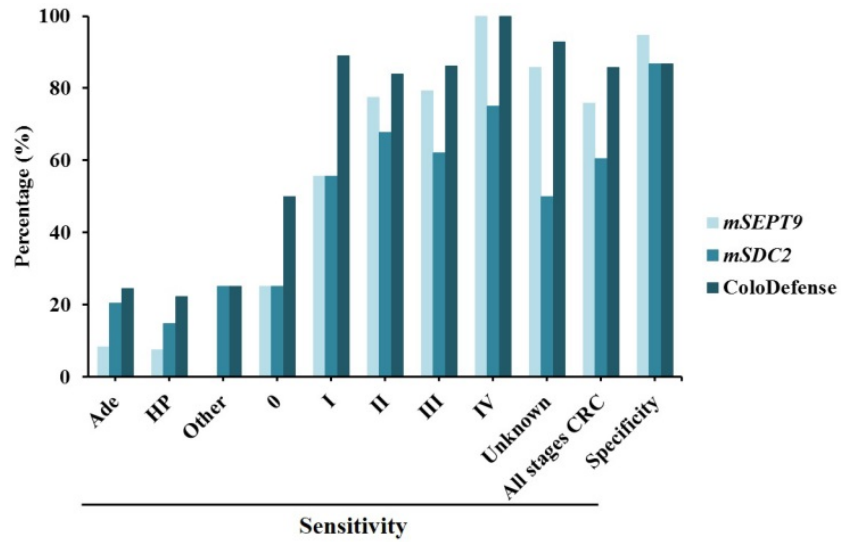

Figure 4. The sensitivities and specificities of plasma-based assay of mSEPT9 alone, mSDC2 alone and ColoDefense test for the detection of Ade, HP, other tumors and different stages of CRC.

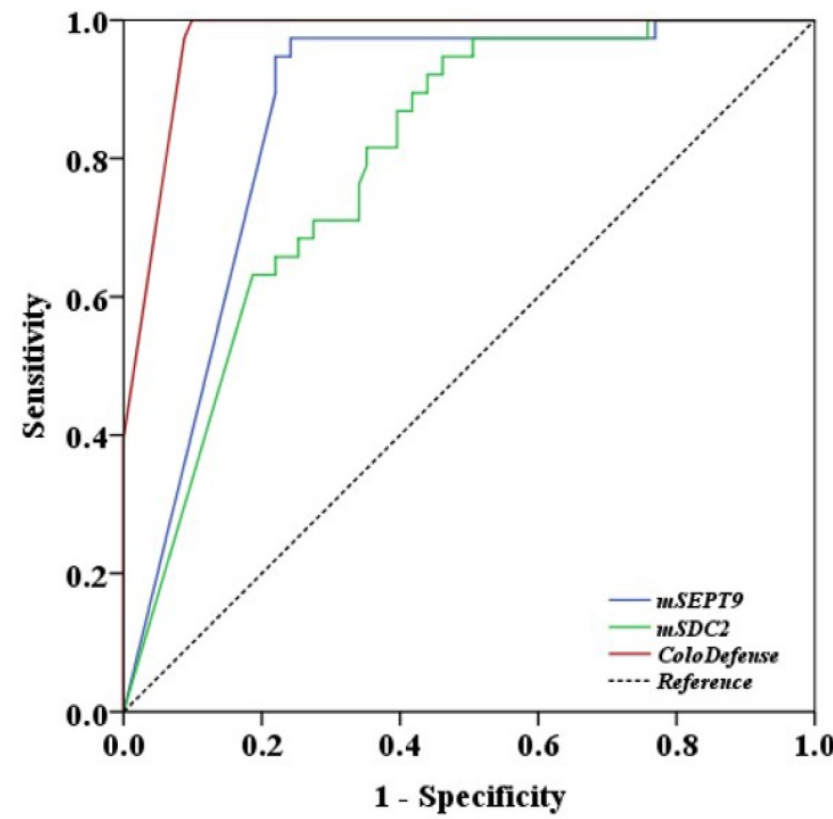

Figure 5. ROC curves for plasma mSEPT9 test alone, mSDC2 test alone and the combined ColoDefense test for detecting CRC.

\section{Discussion}

Ranked as the third most common and the second most deadly cancer type, CRC has attracted growing attention in the past decades [17]. CRC mortality could be largely mitigated if appropriate diagnosis, especially of precancerous lesion, and surveillance were performed in time [18]. Substantially reduced morbidity and mortality rates in the US during the past decade were largely attributed to the high prevalence of CRC screening among adults 50 years and older, as high as $61 \%$ of who have undergone a colonoscopy [19]. However, the invasiveness of colonoscopy, its tedious procedure and relatively high demand for medical resources have resulted in low compliance rate especially among low-to-average risk population in most countries. In this study, a plasma-based multi-target DNA methylation test was shown to be a potential cost-effective solution to this dilemma.

Table 2. Sensitivities of plasma mSEPT9 test alone, mSDC2 test alone and the combined ColoDefense test for detecting CRC of different genders, age groups, stages, tumor sites, tumor sizes and degrees of differentiation.

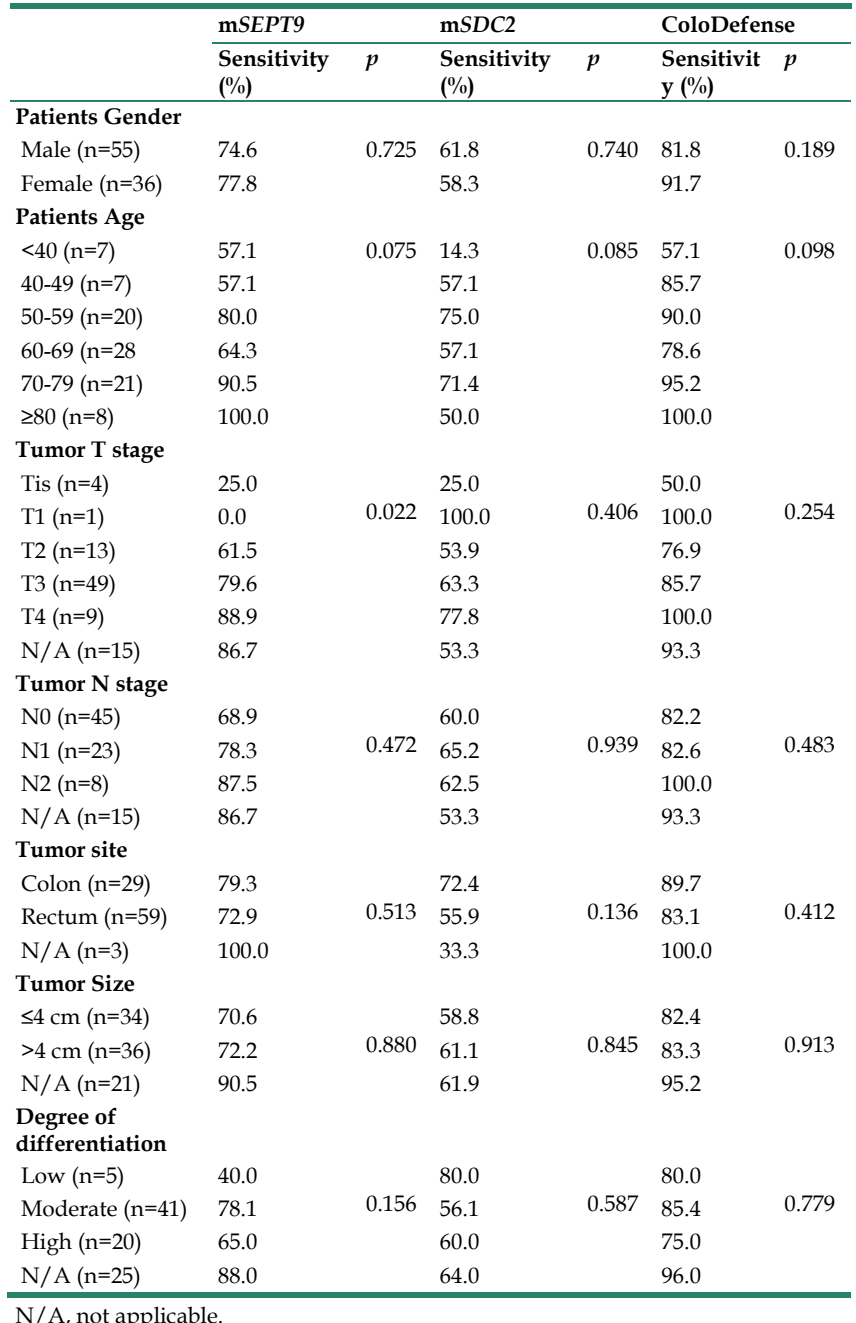

Multiple methylation biomarkers for precancerous lesion and CRC screening have been evaluated independently or in combination in plasma samples. The first blood based mSEPT9 assay approved by FDA, Epi proColon 2.0 assay, exhibited limited sensitivities of $22 \%$ and $68.2 \%$ for precancerous lesions and CRCs, respectively, with a specificity of $78.2 \%$ using $1 / 3$ scoring algorithm [20]. Other retrospective case control studies based on plasma mSEPT9 showed a sensitivity of $\sim 70 \%$ and a specificity of $\sim 90 \%$, while a prospective study in 7941 asymptomic individuals showed a sensitivity of $48.2 \%$ and a specificity of $91.5 \%$ [21, 22]. In our study, the sensitivity of mSEPT9 alone for CRC detection was 
$75.8 \%$ with a specificity of $94.7 \%$, in accordance with previous retrospective studies. $\mathrm{mSDC} 2$ was also suggested as a candidate blood-based biomarker in a panel study, and its performance was evaluated alone or more often when combined with other methylated markers, demonstrating its potential contribution in combination tests $[23,24,9]$. In the present validation study of plasma ColoDefense test, combining mSDC2 with mSEPT9 resulted in an improvement of $9.9 \%$ in sensitivity with a specificity of $86.8 \%$, in good agreement with our previous study that showed $88.9 \%$ sensitivity (95\% CI: $81.4 \%-93.7 \%$ ) and $92.8 \%$ specificity (95\% CI: 87.4\%-96.0\%) in a training set [14]. Therefore, consistent performance of our multiplex DNA methylation test with plasma samples in independent cohorts further validated its potential as an alternative approach for CRC screening.

In general, plasma-based tests for screening are preferred over whole blood mainly because inclusion of blood cells would introduce excessive amount of DNA/RNA as background. Meanwhile, changes in epigenetic characteristics of peripheral blood leukocyte gDNA could directly reflect the status of immune responses triggered by tumor genesis, development, progression and remission. Previous studies of the methylation level of leukocyte gDNA have identified markers that were in concordance with several cancer types or precancerous lesions, including colorectal adenomas, CRC, gastric cancer and breast cancer [25-27]. For example, Zhang et al. showed that the methylation levels of IGFII and N33 and the frequency of mIGFII were significantly higher in gastric cancer cases than those of a high-risk population, suggesting their association with gastric carcinogenesis [28]. Another study showed that a combination of three methylated markers in KIAA1549L and BCL2 genes yielded a c-statistics value of 0.69 in a CRC screening test, less impressive in comparison with other established markers [29]. In this study, no significant differences in the methylation levels of SEPT9 and SDC2 in leukocytes were observed among $\mathrm{CRC}$, Ade, $\mathrm{HP}$ and control groups, while either $\mathrm{mSEPT9}$ or $\mathrm{mSDC} 2$ level alone in plasma were able to distinguish CRC cases from patients with non-cancerous lesions or healthy subjects. These observations indicated that the ratio of certain methylated genes in plasma differed from that in leukocytes, the main proportion of mSEPT9 and $\mathrm{mSDC} 2$ in plasma was originally from cancer tissues rather than leukocytes, and the leukocytes gDNA will not affect the performance of plasma ColoDefense test. Meanwhile, the overwhelming amount of gDNA in leukocytes suggests analyzing methylation levels of candidate genes in both leukocytes and plasma during marker selection to avoid false positive caused by hypermethylation of certain markers only in leukocytes.

Compared with mSEPT9 level in leukocytes, $\mathrm{mSDC} 2$ level was orders of magnitude higher with much lower $\Delta \mathrm{Ct}$ value, suggesting an increased amount of leukocyte $\mathrm{mSDC} 2$ released into plasma that could account for the $7.9 \%$ lower specificity. This further emphasizes that care should be taken to avoid additional blood cell damages when separating plasma and leukocytes. In addition, such a scenario could also account for the optimized $2 / 3$ scoring algorithm for $\mathrm{mSDC} 2$ in comparison to $1 / 3$ algorithm for $\mathrm{mSEPT9}$ as $2 / 3$ algorithm should reduce the interference from accidental rupture of the leukocytes more than $1 / 3$ algorithm.

\section{Conclusion}

In this study, we evaluated the methylation levels of SEPT9 and SDC2 in peripheral blood leukocytes and plasma from patients with CRC and precancerous lesions as well as healthy subjects. Our results showed that the methylation levels of both markers in plasma could differentiate CRC from benign tumors and healthy controls, while their levels in leukocytes could not. Furthermore, the test results indicated that the overall sensitivity for CRC, especially early stage CRC, was greatly improved upon combination of mSEPT9 and $\mathrm{mSDC} 2$. In conclusion, plasma ColoDefense test was a promising tool for CRC early detection.

\section{Acknowledgements}

\section{Funding information}

The work was supported by the grants from the Suzhou Technology Entrepreneur Angel Project (Grant No. CYTS2018051), Key Technologies R \& D Program for Social Development of Jiangsu Province (Grant No. BE2019688), Kunshan Leading Talent Project (Grant No. 00311), Suzhou Innovation and Entrepreneurship Leading Talent Program (Grant No. ZXL2020046) and Key Technologies R \& D Program for Social Development of Xuzhou (Grant No. KC17184).

\section{Authors' contributions}

$\mathrm{ZC}, \mathrm{GZ}$ and $\mathrm{YM}$ performed the statistical analyses and drafted the manuscript. ZC, GZ, KW and $\mathrm{XW}$ participated in sample collection and data analysis. GZ, SX, MZ and SF conceived of the study and participated in the design and coordination of the study. All authors read and approved the final manuscript. 


\section{Ethics approval and consent to participate}

All participants have acknowledged and signed the informed consent, and this study was performed according to the principles of the Helsinki Declaration and approved by the Institutional Review Board of the Affiliated Hospital of Xuzhou Medical University (Ethics Committee reference number: YFY2020KL123-01).

\section{Consent for publication}

Not applicable.

\section{Data availability}

The datasets analyzed during this study are available from the corresponding author on reasonable request.

\section{Competing Interests}

The authors have declared that no competing interest exists.

\section{References}

1. Pantel K, Alix-Panabieres C. Real-time liquid biopsy in cancer patients: fact or fiction? Cancer Res. 2013;73(21):6384-8.

2. Mattox AK, Bettegowda C, Zhou S, Papadopoulos N, Kinzler KW, Vogelstein B. Applications of liquid biopsies for cancer. Sci Transl Med. 2019;11:1-3.

3. Aucamp J, Bronkhorst AJ, Badenhorst CPS, Pretorius PJ. The diverse origins of circulating cell-free DNA in the human body: a critical re-evaluation of the literature. Biol Rev Camb Philos Soc. 2018;93(3):1649-83.

4. Liu X, Ren J, Luo N, Guo H, Zheng Y, Li J et al. Comprehensive DNA methylation analysis of tissue of origin of plasma cell-free DNA by methylated CpG tandem amplification and sequencing (MCTA-Seq). Clin Epigenetics. 2019;11(1):93

5. $\mathrm{Hu}$ Y, Ulrich BC, Supplee J, Kuang Y, Lizotte PH, Feeney NB et al. False-Positive Plasma Genotyping Due to Clonal Hematopoiesis. Clin Cancer Res. 2018;24(18):4437-43

6. Razavi P, Li BT, Brown DN, Jung B, Hubbell E, Shen R et al. High-intensity sequencing reveals the sources of plasma circulating cell-free DNA variants. Nat Med. 2019;25(12):1928-37.

7. Yizhak K, Aguet F, Kim J, Hess JM, Kübler K, Grimsby J, et al. RNA sequence analysis reveals macroscopic somatic clonal expansion across normal tissues. Science. 2019;364(6444).

8. Klutstein M, Nejman D, Greenfield R, Cedar H. DNA Methylation in Cancer and Aging. Cancer Res. 2016;76(12):3446-50.

9. Bartak BK, Kalmar A, Peterfia B, Patai AV, Galamb O, Valcz G et al Colorectal adenoma and cancer detection based on altered methylation pattern of SFRP1, SFRP2, SDC2, and PRIMA1 in plasma samples. Epigenetics. 2017;12(9):751-63.

10. Lamb YN, Dhillon S. Epi proColon ${ }^{\circledR} 2.0$ CE: A Blood-Based Screening Test for Colorectal Cancer. Mol Diagn Ther. 2017;21(2):1-8.

11. Potter NT, Hurban P, White MN, Whitlock KD, Lofton-Day CE, Tetzner R et al. Validation of a real-time PCR-based qualitative assay for the detection of methylated SEPT9 DNA in human plasma. Clin Chem. 2014;60(9):1183-91.

12. Li H, Wang Z, Zhao G, Ma Y, Chen Y, Xue Q et al. Performance of a MethyLight assay for methylated SFRP2 DNA detection in colorectal cancer tissue and serum. Int J Biol Marker. 2019; 34(1): 54-59.

13. Jensen S, Øgaard N, Ørntoft MW, Rasmussen MH, Bramsen JB, Kristensen H et al. Novel DNA methylation biomarkers show high sensitivity and specificity for blood-based detection of colorectal cancer-a clinical biomarker discovery and validation study. Clin epigenetics. 2019;11(1):158.

14. Zhao G, Li H, Yang Z, Wang Z, Xu M, Xiong S et al. Multiplex methylated DNA testing in plasma with high sensitivity and specificity for colorectal cancer screening. Cancer Med. 2019;8(12):5619-28.

15. Zhao G, Ma Y, Li H, Li S, Zhu Y, Liu X et al. A novel plasma based early colorectal cancer screening assay base on methylated SDC2 and SFRP2. Clin Chim Acta. 2020;503:84-9.

16. Oh TJ, Oh HI, Seo YY, Jeong D, Kim C, Kang HW et al. Feasibility of quantifying SDC2 methylation in stool DNA for early detection of colorectal cancer. Clin Epigenetics. 2017;9:126.

17. Ferlay J, Colombet M, Soerjomataram I, Mathers C, Parkin DM, Pineros M et al. Estimating the global cancer incidence and mortality in 2018: GLOBOCAN sources and methods. Int J Cancer. 2019;144(8):1941-53.
18. Winawer SJ, Zauber AG. The advanced adenoma as the primary target of screening. Gastrointestinal endoscopy clinics of North America. 2002;12(1):1-9.

19. Siegel RL, Miller KD, Goding Sauer A, Fedewa SA, Butterly LF, Anderson JC et al. Colorectal cancer statistics, 2020. CA Cancer J Clin. 2020;70(3):145-64.

20. Potter NT, Hurban P, White MN, Whitlock KD, Lofton-Day CE, Tetzner R et al. Validation of a real-time PCR-based qualitative assay for the detection of methylated SEPT9 DNA in human plasma. Clin Chem. 2014;60(9):1183-91.

21. Lamb YN, Dhillon S. Epi proColon(®) 2.0 CE: A Blood-Based Screening Test for Colorectal Cancer. Mol Diagn Ther. 2017;21(2):225-32.

22. Church TR, Wandell M, Lofton-Day C, Mongin SJ, Burger M, Payne SR et al. Prospective evaluation of methylated SEPT9 in plasma for detection of asymptomatic colorectal cancer. Gut. 2014;63(2):317-25.

23. Oh T, Kim N, Moon Y, Kim MS, Hoehn BD, Park CH et al. Genome-wide identification and validation of a novel methylation biomarker, SDC2, for blood-based detection of colorectal cancer. J Mol Diagn. 2013;15(4):498-507.

24. Mitchell SM, Ross JP, Drew HR, Ho T, Brown GS, Saunders NF et al. A panel of genes methylated with high frequency in colorectal cancer. BMC Cancer. 2014;14(54):1-15.

25. Li L, Choi JY, Lee KM, Sung H, Park SK, Oze I et al. DNA methylation in peripheral blood: a potential biomarker for cancer molecular epidemiology. J Epidemiol. 2012;22(5):384-94.

26. Lim U, Flood A, Choi SW, Albanes D, Cross AJ, Schatzkin A et al. Genomic methylation of leukocyte DNA in relation to colorectal adenoma among asymptomatic women. Gastroenterology. 2008;134(1):47-55.

27. Fridley BL, Armasu SM, Cicek MS, Larson MC, Wang C, Winham SJ et al. Methylation of leukocyte DNA and ovarian cancer: relationships with disease status and outcome. BMC Med Genomics. 2014;7(21):1-12

28. Zhang Y, Su HJ, Pan KF, Zhang L, Ma JL, Shen L et al. Methylation status of blood leukocyte DNA and risk of gastric cancer in a high-risk Chinese population. Cancer Epidem Biomar. 2014;23(10):2019-26.

29. Heiss JA, Brenner H. Epigenome-wide discovery and evaluation of leukocyte DNA methylation markers for the detection of colorectal cancer in a screening setting. Clin Epigenetics. 2017;9:24. 